\title{
Assessing the links between resilience, disturbance and functional traits in paleoecological datasets
}

\author{
Rebecca Hamilton', T. Brussel ${ }^{2}$, Q. Asena ${ }^{3}$, R. Bruél ${ }^{4}$, K. Marcisz $^{5}$, M. Słowiński ${ }^{6}$ and J. Morris ${ }^{7}$ \\ 2nd EcoRe3 workshop, Salt Lake City, USA, 8-10 May 2018
}

Functional traits are integral to understanding the patterns and drivers of ecological resilience. Response traits determine whether a system exhibits properties resulting in higher resistance or faster recovery rates, while effects traits are important for identifying links between environmental changes and ecosystem functioning. Although paleoecological research methods have been identified as an important tool for understanding patterns of ecological resilience across different regions, paleoecologists have only recently begun to think about how functional ecological approaches can be used in the context of sub-fossil assemblages from sediments. Thus, the second EcoRe 3 workshop aimed to (i) explore developments in modeling ecosystem resilience from the paleorecord, and (ii) investigate the development of new tools to link paleoecological datasets with functional trait databases derived from neo-ecological research.

These two themes were investigated over two days at the Natural History Museum of Utah in the form of presentations and semistructured discussion groups. Twenty-two participants from nine countries attended the meeting, with funding provided by PAGES and the Research Council of Norway. Research covered in the meeting was diverse with respect to the ecosystems studied, proxies (pollen, Cladocera, testate amoebae, diatoms) and timescales interrogated, and modeling methodologies applied. The diversity of approaches highlighted advancements the working group had made in measuring longterm patterns of ecosystem resilience since the last meeting.

Presentations themed around modeling resilience from long-term ecological data showcased progress in the development of novel techniques for measuring critical transitions, and presented particular records that serve as interesting case studies for the exploration of aspects of resilience. They highlighted the value of high-resolution data (taxonomic and temporal) that capture ecological regime shifts and/or clearly identifiable drivers of change for examination of ecological thresholds and response rates.

Further progression in using paleoecology for measuring resilience requires improvements in the capacity of associated research to capture individual and collective functional traits that can be related to the resistance or recovery rate of an ecosystem following disturbance. This is particularly apparent where no critical thresholds are breached, when there is a leador lag-proxy response to drivers of change, or when multiple stressors operating at different time scales are involved. Several presentations therefore sought to explore these issues via (1) case-studies focused on the role that particular traits play in resistance to, or recovery from, disturbance, and (2) demonstrating techniques for measuring changes in functional trait space through time, and linking these to ecological resilience.

While these presentations elucidated the importance of response and effect traits in controlling ecosystem function, it became apparent that there are several hurdles to overcome to progress the field of functional paleoecology and resilience. A horizon scan of key issues pertaining to what these challenges comprise, and how they can be resolved, was the key focus of the workshop discussion groups (Fig. 1). Identifying which traits are both ecologically meaningful and quantifiable in the sediment record was highlighted as a major challenge for many microfossil types. Linked to this are issues related to differential (or low) taxonomic resolution, and quantification of drivers that require the development of a unique set of analytical approaches if functional ecological approaches are applied to paleoecological data. The final discussions centered on the potential to advance understanding related to conservation outcomes if these technical challenges can be overcome. An in-depth analysis of these challenges and the solutions required to overcome them form the basis of an upcoming EcoRe3 perspective paper.

The workshop concluded with a discussion regarding EcoRe3's future steps, including (1) the publication of a special issue based on the overall goals in 2019, and (2) regrouping the members for a themed session at INQUA 2019 in Dublin, Ireland. A follow-up workshop, focused around the statistical modeling of paleo datasets using techniques developed within EcoRe3, was also proposed. Details of future EcoRe 3 activities will be updated on the website (pastglobalchanges.org/ini/wg/ecore3) and on the following social media outlets.

- EcoRe3 on Facebook (visit page and request to join): facebook.com/ groups/286999515057710

-EcoRe3 on Twitter: @Eco_Re3

- EcoRe3 on ResearchGate: researchgate. net/project/EcoRe3-Resistance-Recoveryand-Resilience-in-Long-term-EcologicalSystems-2

\section{AFFILIATIONS}

'School of Culture, History \& Language, The Australian National University, Canberra, Australia

${ }^{2}$ Geography Department, University of Utah, Salt Lake City, USA

${ }^{3}$ School of Environment, University of Auckland, New Zealand

${ }^{4}$ UMR CARRTEL, INRA-Université Savoie Mont-Blanc, Thonon-les-bains, France

${ }^{5}$ Laboratory of Wetland Ecology and Monitoring and Department of Biogeography and Palaeoecology, Adam Mickiewicz University, Poznań, Poland

Department of Environmental Resources and Geohazards, Polish Academy of Sciences, Warsaw, Poland

'Research Development Office, University of Utah, Salt Lake City, USA

CONTACT

Rebecca Hamilton: rebecca.hamilton@anu.edu.au

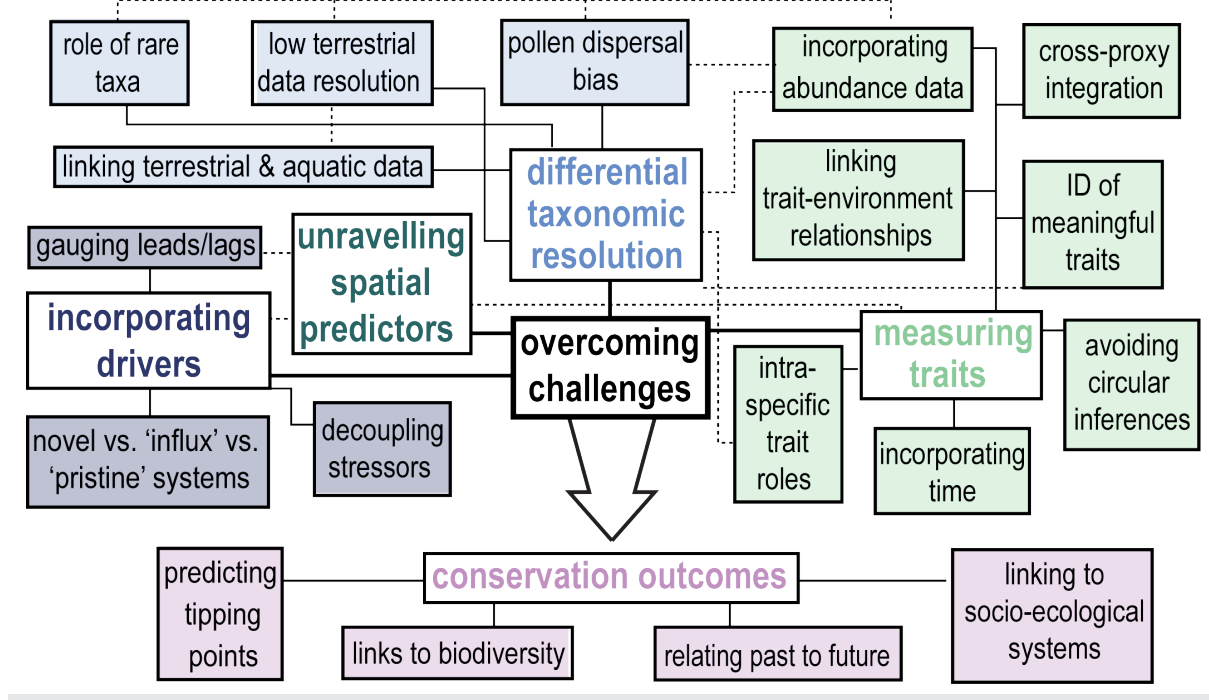

Figure 1: Key challenges to be resolved in paleoecology related to function and measuring resilience to advance conservation outcomes. 